AperTO - Archivio Istituzionale Open Access dell'Università di Torino

\title{
Association between maternal education and survival after childhood cancer
}

\section{This is the author's manuscript}

Original Citation:

Availability:

This version is available http://hdl.handle.net/2318/1690701

since 2020-05-08T10:45:50Z

Published version:

DOI:10.1002/pbc.27616

Terms of use:

Open Access

Anyone can freely access the full text of works made available as "Open Access". Works made available under a Creative Commons license can be used according to the terms and conditions of said license. Use of all other works requires consent of the right holder (author or publisher) if not exempted from copyright protection by the applicable law. 


\title{
Association between maternal education and survival after childhood cancer
}

\author{
Isaevska $\mathrm{E}^{1}$, Popovic $\mathrm{M}^{1}$, Alessi $\mathrm{D}^{1,2}$, Mosso $\mathrm{ML}^{1}$, Sacerdote $\mathrm{C}^{1}$, Magnani $\mathrm{C}^{3}$, Pastore $\mathrm{G}^{1}$, Rosso $\mathrm{T}^{1}$, Zengarini $\mathrm{N}^{4}$, \\ Dockerty $\mathrm{J}^{5}$, Merletti $\mathrm{F}^{1}$, Maule $\mathrm{M}^{1}$. \\ 1 \\ Childhood Cancer Registry of Piedmont, Cancer Epidemiology Unit, Department of Medical Sciences, \\ University of Turin, Turin, Italy. \\ 2 \\ Piedmont Cancer Registry, Biella and Vercelli Provinces, Epidemiology Unit, ASL VC, Vercelli, Italy. \\ Department of Translational Medicine, Unit of Medical Statistics and Cancer Epidemiology, CPO Piemonte \\ and University of Piemonte Orientale, Novara, Italy. \\ Epidemiology Unit, ASL TO3 Piedmont Region, Grugliasco, Italy. \\ 5 \\ Department of Preventive and Social Medicine, University of Otago, P O Box 56, Dunedin, New Zealand.
}

\section{Abstract \\ Background}

Several nonbiological factors, including socioeconomic status indicators and other family characteristics, influence survival from childhood cancers. Our study explores the association between parental education and childhood cancer survival.

\section{Methods}

The specialized Childhood Cancer Registry of the Piedmont region in Italy provided data on all the cases (aged 0-14) diagnosed with cancer in the period 1976-2011 who resided in the city of Turin (capital of the Piedmont region) at least once since 1971. Information on parental education was extracted from the Turin Longitudinal Study by record linkage. The association between parental educational level and survival was estimated using Cox regression.

\section{Results}

The study included 949 children. We observed a disadvantage in the overall survival for children of less educated mothers. No such effect was observed for paternal education. The effect of maternal education was particularly strong for central nervous system tumors (hazard ratios, $2.9 ; 95 \%$ confidence interval, 1.1-8.0). A similar effect, though smaller in magnitude, was observed for leukemia and embryonal tumors, whereas the estimates for lymphoma were imprecise.

\section{Conclusions}

Our study shows an association between maternal educational level and survival in children with central nervous system tumors, a diagnosis that often requires long lasting treatment and special care. Giving support to the families of affected children to provide them the optimal care has the potential to improve children's cancer treatment outcomes. 


\section{Abbreviations}

- CCRP

- Childhood Cancer Registry of Piedmont

- CI

- confidence interval

- $\mathrm{CNS}$

- central nervous system

- DCO

- death certificate only

- HR

- hazard ratio

- ICCC

- International Classification of Childhood Cancer

- IQR

- interquartile range

- SES

- socioeconomic status

- TLS

- Turin Longitudinal Study

\section{INTRODUCTION}

Several socioeconomic status (SES) indicators and family characteristics have been found to influence survival from childhood cancer, 1 independent of biological predictors of survival and despite the accessibility of modern diagnostic techniques and treatments within countries that provide universal health care.

An association between poorer childhood cancer survival and lower SES indicators was observed in both low income and high income countries. 1 Several studies, $2-\underline{5}$ mainly conducted in Northern European countries, have found an association between parental education and childhood cancer survival. This association was not observed for household income, $\underline{2}, \underline{3}$ indicating the possibility that parental education might not be a proxy of material resources, but rather of other, nonmaterial, assets of the parents. Survival after childhood cancer also showed an association with several family characteristics such as the number of siblings, $\underline{2}, \underline{4}, \underline{6}, \underline{7}$ cohabitation status of parents, $\underline{4}$ and parental age. $\underline{6}, \underline{7}$ Lower survival in children with less educated parents, single parent families, and families living far from the treatment center was also observed in a study from Greece $\underline{8}$ that included only leukemia cases. The authors argued that these associations may indirectly reflect how family workload, ability to rely on help from other family members, and maturity of the parents to cope with the diagnosis may influence survival.

The effect of parental education on survival appears to vary for different tumor types, with the strongest effects on central nervous system (CNS) tumors, leukemias, and tumors with long lasting treatments in general. $\underline{2}, \underline{5}$

Our study uses data from the Childhood Cancer Registry of Piedmont, a region in North West Italy with an oncologic referral center belonging to the national network of the Italian Association of Pediatric Hematology Oncology centers of excellence. By virtue of the Italian universal health care setting, access to and quality of health care are warranted free of charge to all children, independently of different levels of family income and economical resources. Our objective was to 
evaluate the association between parental education and overall survival after cancer diagnosis in children, and to verify if this association depends on the tumor type. This study, together with two Greek studies, $\underline{8}, \underline{9}$ is one of the first on this topic in the Southern European population.

\section{MATERIALS AND METHODS}

\subsection{Study population}

Data on childhood cancer cases (diagnosed between the ages 0 and 14) were collected from the Childhood Cancer Registry of Piedmont (CCRP), whereas data on parental education were retrieved from The Turin Longitudinal Study (TLS).

\subsection{The CCRP}

Since 1967, CCRP has collected information on all incident malignant neoplasms and benign intracranial neoplasms in all children up to 14 years of age (and since 2000 also in adolescents, 15 to 19 years old) who are residents of Piedmont, a region with approximately 4.5 million inhabitants in North West Italy. Before availability of the hospital admission and discharge files (from the mid 90s), cases were searched through manual perusal of hospital registries and clinical records. From then on, cases are identified from the hospital admission and discharge files, including hospital stays of Piedmont residents in all Italian hospitals. The potentially relevant discharge diagnoses are then selected, and their complete clinical documentation is requested and examined by an experienced pediatrician in order to identify incident cases. Further case ascertainment is obtained from the databases of pathology departments of Piedmont hospitals, records for reimbursements for cancer treatments administered abroad, the Turin Cancer Registry and the database of the Italian Association of Pediatric Hematology and Oncology. Only CCRP authorized personnel can access individual data. Data are anonymized before statistical analysis.

Cases are included in the CCRP only after confirmation of their age and residence in Piedmont at the time of diagnosis by the Town Registrar Office. The registered information includes personal, clinical, and follow up data. All diagnoses are coded using the ICD O 3 classification 10 and grouped into the diagnostic groups of the International Classification of Childhood Cancer (ICCC 3).11 CCRP has high quality data according to the usual indicators, such as microscopic verification of diagnosis (93.0\%) and cases identified through death certificate only (1.1\%).12 The CCRP personnel periodically (every 2-3 years) updates the vital status of the registered cases using information from the Registrar Offices of the town of residence (within and outside Piedmont, should the patients emigrated in another Italian region). Vital status was last updated on December 31, 2012. From 1967 to 2011, CCRP recorded 4411 incident childhood cancer cases aged 0 to 14 years at diagnosis. CCRP includes demographic information (name, date, and place of birth) on the legal parents, biological or adoptive, of all registered children.

\subsection{The TLS}

The TLS 13 includes persons who have resided in the city of Turin at least once since 1971, based on information from the historical population registries of the Town Registrar Offices.

Demographic data of this cohort are integrated with socioeconomic information from the decennial Italian censuses $(1971,1981,1991$, and 2001). The long study period, the several sources of information on parental education level (four censuses and the historical population registry), not always consistent, and the 10 year period between censuses did not allow us to verify the parental educational level at the exact time of the diagnosis. In order to minimize misclassification of 
parental education level, we decided to use the highest education level ever achieved and to categorize it as high (university degree or upper secondary school) and low (lower secondary school, vocational qualification, elementary school or illiterate, typically achieved at 14 years of age).

In order to exclude cases diagnosed in the period when the survival for most tumor types was very poor, $14, \underline{15}$ we restricted the analyses to the period 1976-2011, accounting for a total of 3667 incident cases. In this study period, 949 CCRP cases and their parents, residing in the city of Turin at some point since 1971, were successfully linked with TLS. The record linkage was performed using name, gender, date and place of birth, date and place of residence at the moment of diagnosis of the child, name of her/his parents and their dates and places of birth. As expected, successful linkage did not always mean that the education level of both parents was available.

\subsection{Statistical analysis}

Our outcome was the overall survival. Children were followed up from the time of diagnosis until the date of death from any cause, emigration abroad (five cases emigrated or were lost to follow up in the period 1976-2012), or end of the last follow up (December 31, 2012), whichever occurred first. Cases identified by death certificate only (DCO) were included in the descriptive statistics, but were excluded from further analyses $(N=4$, period 1976-2011). After excluding cases with missing information on parental education and/or age, the total number of children included in the complete case analysis was 709 (75\% of the total). The characteristics of the children included in the complete case analysis and the characteristics of the cases that were excluded due to missing information (on parental educational level and/or parental age and DCO cases with survival time zero) are shown in Table S1 in the Supporting Information. Kaplan-Meier curves were used to estimate the crude survival proportion of children in the two levels of parental education in two time periods (1976-1995 and 1996-2011) to account for the strongly increasing survival trends in the last decades. Differences between the survival curves for low and high education within the two periods of diagnosis were tested using the log rank test. 16

The association between parental education level and survival was estimated as hazard ratios (HRs) and $95 \%$ confidence intervals $(95 \% \mathrm{CI})$ using Cox regression models, and the proportional hazards assumption was tested using Schoenfeld residuals. The model included maternal education, paternal education, and year of diagnosis. The latter strongly affects both survival and the proportion of parents with higher levels of education and was included in the model as a linear variable. To evaluate whether associations of parental education with child survival are driven by parental maturity and life experience, we performed additional analyses by including in the models maternal and paternal ages at diagnosis in the model as continuous variables.

Assuming that the effects of parental education may be different for different malignancies, we conducted separate analyses for leukemia, lymphoma, CNS tumors, and embryonal tumors. The group of embryonal tumors includes all ICD O 3 morphological codes used in a previous CCPR study, 12 excluding the group of intracranial and intraspinal embryonal tumors (included in the group of CNS tumors). In order to understand whether the effect of parental education on survival could be due to earlier recognition of symptoms and, therefore, diagnosis at earlier stages, a post hoc analysis on CNS tumors was additionally adjusted for the stage at diagnosis (coded as local vs regional/metastatic). Because the percentage of missing data after the linkage with the TLS was rather high for maternal and paternal education ( $20 \%$ and $11 \%$, respectively), we performed multiple imputations by the technique of chained equations, 17 assuming missing at random data. The variables without missing values included in the imputation procedure were the following: year of diagnosis, follow up duration, failure event, and tumor type. Maternal and paternal education were imputed as binary variables, whereas maternal and paternal age at diagnosis, with $1 \%$ and $2 \%$ 
of missing data, respectively, were imputed as continuous variables. The number of imputations was set to 20. All the analyses were performed using STATA version 13 (STATA Corp., Texas, USA). 18

\section{RESULTS}

Out of 949 children diagnosed with cancer in the period 1976-2011, who resided in Piedmont at the time of diagnosis and were successfully linked with the TLS data, $306(32 \%)$ were diagnosed with leukemia, $217(23 \%)$ with a CNS tumor, $157(17 \%)$ with an embryonal tumor, and $123(13 \%)$ with lymphoma (Table 1$)$. There were 316 deaths (33\% of the total cancer cases) during the period of follow up. The median follow up time was 10 years (interquartile range, 2.2-23.5). In the first diagnostic period (1976-1995), around 23\% of the children had highly educated mothers, whereas in the last diagnostic period (1996-2011), that percentage reached 52\%. For the two diagnostic periods, the percentage of fathers with the higher level of education was $34 \%$ and $50 \%$, respectively. Mean parental age at diagnosis was 36.2 (SD, 6.8) and $39.6(\mathrm{SD}, 7.3)$ for mothers and fathers, respectively.

Table 1. Main characteristics of the cases (0-14 years) from the CCRP, diagnosed between 1976 and 2011 and linked with the TLS

\section{No of casesa $($ mean \pm SD or \%)}

Total

Maternal education

$\begin{array}{ll}\text { High } & 260(27.4) \\ \text { Low } & 499(52.6) \\ \text { Missing } & 190(20.0)\end{array}$

Paternal education

$\begin{array}{ll}\text { High } & 337(35.5) \\ \text { Low } & 503(53.0) \\ \text { Missing } & 109(11.5) \\ \begin{array}{l}\text { Maternal age at } \\ \text { diagnosis } \\ \text { Missing }\end{array} & 940(36.2 \\ \text { Paternal age at diagnosis } & 929(39.6) \\ \text { Missing } & 20(2.1) \\ \text { Tumor type } & \\ \text { Leukemia } & 306(32.2) \\ & 123(13.0) \\ \text { Lymphoma } & 217(22.9)\end{array}$

\section{Deathsa (\%) PY Median (IQR)}

316

$12498.4 \begin{aligned} & 10.0(2.2- \\ & 23.5)\end{aligned}$

$\begin{array}{lll}58(18.4) & 2961.7 & 8.5(2.8-17.6) \\ 211(66.8) & 6587.3 & 8.9(1.6-25.7) \\ & & 14.3(5.0- \\ 47(14.9) & 2949.5 & 24.7)\end{array}$

\begin{tabular}{|c|c|c|}
\hline 99 (31.3) & 4107.1 & $9.3(2.6-19.4)$ \\
\hline $190(60.1)$ & 6711.1 & $9.6(1.8-25.5)$ \\
\hline $27(8.5)$ & 1680.2 & $\begin{array}{l}14.6(4.2- \\
24.7)\end{array}$ \\
\hline $\begin{array}{l}314(35.9 \pm \\
6.9)\end{array}$ & 12471.5 & $\begin{array}{l}10.3(2.3- \\
23.8)\end{array}$ \\
\hline $2(0.6)$ & 26.9 & $2.0(1.4-2.4)$ \\
\hline $\begin{array}{l}310(39.6 \pm \\
7.6)\end{array}$ & 12331.7 & $\begin{array}{l}10.3(2.3- \\
23.8)\end{array}$ \\
\hline $6(1.9)$ & 166.7 & $4.3(1.9-12$. \\
\hline
\end{tabular}


No of casesa $($ mean \pm SD or $\%$ )

Embryonal tumors $157(16.5)$

Other tumor types $146(15.4)$

Child's age at diagnosis

$\begin{array}{ll}0-4 & 360(37.9) \\ 5-9 & 264(27.8) \\ 10-14 & 325(34.2)\end{array}$

Child's gender

Male

Female

$419(43.8)$

Period at diagnosis

1976-1995 $574(60.5)$

1996-2011 $375(39.5)$
Deathsa $(\%) \quad$ PY Median (IQR)

$54(17.1) \quad 2063.9 \quad 7.8(2.2-25.7)$

$60(19.0) \quad 1858.2 \quad 9.8(2.1-22.8)$

$109(34.5) \quad 4555.8 \quad 8.8(2.3-22.1)$

$94(29.8)$

$3622.5-11.1(2.2-$

25.5)

$114(35.8) \quad 4320.1 \quad 10.5(2.1-$

23.0)

$\begin{array}{lll}178(56.3) \quad 7138.4 & 10.5(2.4- \\ 24.0)\end{array}$

$138(43.7) \quad 5360.1 \quad 9.8(2.2-22.5)$

$\begin{array}{lll}258(81.6) \quad 9590.7 & 19.8(1.6- \\ 28.7)\end{array}$

$58(18.4) \quad 2907.7 \quad 7.5(2.9-12.3)$

- CNS, central nervous system; IQR, interquartile range; PY, person years; SD, standard deviation.

- ${ }^{a}$ The number of cases and deaths includes DCO cases $(N=4)$.

Among children affected by any tumor type, the favorable effect of having a mother with higher education can be seen in Figure 1A and 1B. This effect is apparent in both, 1976-1995 and 19962011, diagnostic periods ( $\log$ rank test for equality of survivor functions $P=0.006$ and $P=0.020$, respectively). The effect of paternal educational level on survival was smaller (Figure $\underline{1} \mathrm{C}$ and $\underline{1} \mathrm{D}$ ).

\section{Figure 1}

Open in figure viewerPowerPoint

Kaplan-Meier survival estimates according to parental educational level in two diagnostic periods (1976-1995 and 1996-2011) for all tumor types combined. The differences between the parental education levels were tested using the log rank test for homogeneity. The complete case dataset was used

The associations of parental education with survival from childhood cancer from all tumor types combined are shown in Table $\underline{2}$, whereas the same associations stratified by tumor type (leukemia, lymphoma, CNS tumors, and embryonal tumors) are shown in Table $\underline{3}$. All the models met the proportional hazards assumption. As the effect of parental age on the child's survival changed out estimates only marginally, the analyses were adjusted only for the year of diagnosis and mutually adjusted for maternal and paternal education level. The results of the complete case analysis were similar to those after multiple imputation of missing data. For all tumor types combined, the survival was poorer in children of less educated mothers, with the HR of 1.56 (95\% CI, 1.10-2.22; multiple imputation analysis). There was no significant difference in survival related to fathers' education (HR, 0.91). 
Table 2. Parental education and survival after childhood cancer for all tumor types combined

\section{Complete case analysis Multiple imputation}

\section{D/C HR (95\% CI) a HR (95\% CI) a}

Maternal education

$\begin{array}{llll}\text { High } & 47 / 234 & 1.00 & 1.00 \\ \text { Low } & 203 / 475 & 1.71(1.18-2.47) & 1.56(1.10-2.22)\end{array}$

Paternal education
High
$77 / 2631.00$
1.00
Low
$173 / 4460.91(0.68-1.23) 0.91(0.69-1.20)$

$$
0.95(0.94-0.97) 0.95(0.94-0.97)
$$

Year of diagnosis

- $\mathrm{CI}$, confidence interval; $\mathrm{D} / \mathrm{C}$, number of deaths/number of cases in the corresponding category; HR, hazard ratio.

- ${ }^{a}$ The HRs are adjusted for year of diagnosis and mutually adjusted for maternal and paternal education.

Table 3. Parental education and survival from leukemia, lymphoma, CNS tumors and embryonal tumors

\section{Leukemia}

\section{Complete case analysis \\ HR $(95 \%$ \\ $\begin{array}{cc}\text { D/C } & \text { HR }(95 \% \\ \text { CI })\end{array}$}

Multiple imputation
Complete case analysis

Lymphoma

Maternal education

\begin{tabular}{|c|c|c|c|c|c|}
\hline High & $17 / 79 \quad 1.00$ & 1.00 & $8 / 25$ & 1.00 & 1.00 \\
\hline Low & $70 / 155 \begin{array}{l}1.53(0.84- \\
2.76)\end{array}$ & $1.43(0.80-2.54)$ & $18 / 65$ & $\begin{array}{l}0.60(0.20- \\
1.80)\end{array}$ & $0.67(0.23-1.96)$ \\
\hline
\end{tabular}

Paternal education

\begin{tabular}{|c|c|c|c|c|c|c|}
\hline High & $31 / 95$ & 1.00 & 1.00 & $8 / 29$ & 1.00 & 1.00 \\
\hline Low & $56 / 139$ & $\begin{array}{l}1.04(0.65- \\
1.67)\end{array}$ & $1.03(0.65-1.64)$ & $18 / 61$ & $\begin{array}{l}1.30(0.43- \\
3.90)\end{array}$ & $1.36(0.48-3.84)$ \\
\hline $\begin{array}{l}\text { Year of } \\
\text { diagnosis }\end{array}$ & & $\begin{array}{l}0.94(0.92- \\
0.97)\end{array}$ & $0.94(0.92-0.97)$ & & $\begin{array}{l}0.95(0.91- \\
0.99)\end{array}$ & $0.94(0.90-0.98)$ \\
\hline
\end{tabular}

CNS tumors
Complete case analysis
D/C HR (95\% CI)

Multiple
imputation
HR $(95 \%$ CI)

\section{Embryonal tumors}

Complete case analysis

Multiple imputation
D/C HR (95\% CI)
HR (95\% CI)

Maternal education

\begin{tabular}{|c|c|c|c|c|c|}
\hline High & $5 / 46 \quad 1.00$ & 1.00 & $9 / 50$ & 1.00 & 1.00 \\
\hline Low & $50 / 118 \begin{array}{l}3.31(1.18- \\
9.26)\end{array}$ & $2.93(1.08-7.96)$ & $25 / 61$ & $\begin{array}{l}1.75(0.62- \\
4.89)\end{array}$ & $1.57(0.65-3.76)$ \\
\hline
\end{tabular}

Paternal education

\begin{tabular}{|c|c|c|c|c|}
\hline High & $13 / 51 \quad 1.00$ & 1.00 & $11 / 531.00$ & 1.00 \\
\hline Low & $42 / 1130.67(0.31-$ & $0.72(0.38-1.38)$ & $23 / 58 \quad 1.30(0.51-$ & $0.93(0.44-2.00)$ \\
\hline
\end{tabular}


Leukemia

\section{Complete case analysis HR (95\% \\ $\begin{array}{rr}\text { D/C } & \text { HR ( } 95 \%\end{array}$}

1.31)

\section{Multiple imputation \\ HR (95\% CI)}

\author{
Lymphoma \\ Multiple \\ imputation \\ D/C HR (95\% CI) \\ $0.99(0.95-$ \\ HR (95\% CI) \\ $0.98(0.95-1.01)$
}

$0.95(0.92-$ $0.98)$

$0.95(0.92-0.98)$

Year of diagnosis

- $\mathrm{CI}$, confidence interval; $\mathrm{D} / \mathrm{C}$, number of deaths/number of cases in the corresponding category; HR, hazard ratio.

- All HRs are adjusted for year of diagnosis and mutually adjusted for maternal and paternal education.

We observed lower survival for children of less educated mothers for most tumor types, except for lymphoma (Table 3 ). The strongest association was observed in the group of CNS tumors (multiple imputation HR, 2.93; 1.08-7.96) - a three fold increased hazard of death in children whose mothers had low educational level compared with those with at least upper secondary school. With additional adjustment for CNS tumor stage, the estimates changed only marginally (maternal educational level HR, 2.98 (95\% CI, 1.06-8.36); local versus regional/metastatic tumor at diagnosis HR, 1.64 (95\% CI, 0.95-2.84).

Overall, childhood cancer survival did not seem to be associated with paternal educational level (Table $\underline{2}$, the imputation HR for low paternal education was 0.91 ). When stratified by tumor type, paternal education appeared to have no effect on survival of children diagnosed with leukemia or embryonal tumors; there was a very small association between paternal lower education and poorer survival after lymphoma, whereas the opposite was observed after a CNS tumor. However, these estimates are accompanied by wide CIs.

\section{DISCUSSION}

We found an association between the level of maternal education and survival after childhood cancer, with shorter survival in children with less educated mothers (lower secondary school diploma or less). The strongest association was observed in children diagnosed with a CNS tumor, where both the direction and the magnitude of the association were robust after accounting for the stage at diagnosis. Similar associations were observed for survival after leukemia and embryonal tumors. Paternal education showed no clear association with childhood cancer survival.

The estimates for lymphoma are imprecise, with associations between parental education and survival in the opposite direction compared with those seen in other tumor types. Cautious interpretation is necessary, because the number of observations in this group is rather small and the CIs wide.

Studies conducted in European high income countries that, like Italy, have a universal health care system, often observed an association between higher parental education and better survival after childhood cancer.

A nationwide Swedish cohort study $\underline{3}$ showed worse survival in children of parents without post secondary education (based on maternal education when available, otherwise on paternal), 
whereas income was not associated with survival. A Norwegian study $\underline{2}$ also reported that survival was not affected by parental earnings, whereas in the category of tumors that need a long lasting treatment (CNS tumors, leukemias, neuroblastoma, and bone cancers), lowest mortality was seen for children whose mothers had tertiary education, and in those without siblings. In a Swiss study, $\underline{5}$ both high maternal and paternal education were associated with lower cancer mortality. In this study, the stratified analysis by tumor type showed the strongest effect for CNS tumor patients, with survival reduced to half in children of less educated parents. A study from Greece $\underline{8}$ on childhood leukemia reported a better survival in children whose mothers had more than 10 years of schooling, were married, and lived close to the treating center. Another Greek study $\underline{9}$ observed better survival after leukemia diagnosis in children whose parents had higher socio professional background, whereas maternal education showed no association.

Two Danish studies $\underline{4}, \underline{7}$ found no strong effect of parental education on child survival from CNS tumors. In the first study, $\underline{4}$ the survival from all tumor types was only slightly associated with parental education and income, whereas better survival was found when parents were living together, and worse survival when the child had siblings.

Our study is the first in Italy and one of the very few conducted in Southern Europe to estimate the effect of parental education on the survival of children with cancer. We used high quality population based registry data that allow precise calculation of survival time, and we had the opportunity to enrich these data with information from a longitudinal study on socioeconomic determinants of health.

However, our study has several limitations. The small sample size in some categories, such as lymphoma, was insufficient to provide interpretable results. For the same reason, we could not stratify for tumor subgroups, in particular in the category of embryonal tumors, which is a group of several malignancies with different morphology, clinical presentation, treatment options, and prognosis. Also, the possible effect of other SES indicators on childhood cancer survival was not explored in this study, due to lack of information.

Another critical point is the dichotomization of parental education level into high (university degree or upper secondary school) and low (lower secondary school). The reason for this apparently oversimplified choice was three fold: (1) to maximize the number of subjects in the groups, (2) to avoid exposure misclassification due to the lack of information at the exact time of diagnosis (censuses are available only every 10 years). Because at diagnosis, all parents were above 19 (when upper secondary school is generally achieved in Italy), even if they were still pursuing higher qualifications (beyond upper secondary school), this would have not changed their education level (already classified as high in our analysis), (3) to account for the secular trend of increasing education level given that our study period ranges from the 1970s (when university degree was a rather rare educational attainment) to modern days.

Finally, we assumed that the data are missing at random. However, we cannot exclude that this assumption required by the imputation method used was satisfied, possibly biasing our estimates.

Our findings agree with other studies that have observed an effect of parental education level on childhood cancer survival. Parental education could be interpreted as a proxy of the knowledge related assets of the parents, and not as much as a measure of material wealth in settings where health care for children with cancer is funded by taxpayers.

More educated mothers may have healthier children due to healthier life style and higher health care literacy. However, in the context of caring for a child with a cancer diagnosis, higher maternal 
education may indicate better capacity to cope with the child's diagnosis, and to adapt to the emotional and time consuming requirements of adherence to long term care.

The observed associations involved maternal rather than paternal education and were stronger for some tumor types. Leukemias, CNS tumors, and some types of embryonal tumors usually require long lasting treatment and follow up care. In particular, due to the location of the cancer and its aggressive therapy, children with CNS tumors often suffer from impaired cognitive function and have lower quality of life 19 in comparison with children with other tumor types, further loading the burden of the primary caregiver, usually the mother.

The possible mechanisms linking parental education with childhood cancer survival are complex and possibly related with other nonbiological determinants of survival. 1 Maternal education may affect survival after childhood cancer in many ways. Better education might imply better jobs and thus the possibility to reduce working hours, allowing better adaptation to the requirements of inpatient and outpatient care during lengthy treatments. The Italian Federation of Childhood Cancer Parent Associations reported that one of the major sources of distress during their child's hospitalization comes from managing their working commitments and everyday life. 20 Studies suggest that the number of children in the family (often negatively associated with maternal education) and the cohabitation status of the parents might also influence survival. $\underline{2}, \underline{4}, \underline{6}-\underline{8} \mathrm{We}$ did not have information on the actual family composition or longitudinal data on the cohabitation status of the child and his/her parents, and we were thus not able to investigate if this could modify the association between parental education and survival.

Parental education may also influence survival through delayed diagnosis and advanced cancer stage at presentation.21 We attempted to assess the role of the stage at diagnosis for CNS tumors by including it in our model but these only marginally changed our results.

Despite consistent association with childhood cancer survival, socioeconomic determinants are often neglected by policy makers and the health care systems. Insufficient parental understanding of diagnosis, medical instructions and advice, can hamper active participation and continuous involvement in the long process of child treatment. Good communication with the health care staff, psychological and practical support (for instance, help in attending daily duties and caring for the other children, enforcement of laws that grant parents full leave of absence during the care of a severely sick child) may improve adherence to therapy and increase survival. To our knowledge, intervention studies assessing the full potential of this integrated approach have not been conducted.

In conclusion, we found that maternal education may influence survival after childhood cancer. Whatever the underlying mechanisms, this risk factor may be easily modifiable. Giving support to the families of affected children, improving their knowledge on the course of the disease and its treatments, and helping them provide the optimal care has the potential to improve children's cancer treatment outcomes.

\section{ACKNOWLEDGMENTS}

This research was supported by the Master of Epidemiology (University of Turin). E.I. received scholarship from the European Commission through the Erasmus Mundus for Western Balkans II programme, Grant Agreement 2011-2586/001 001 EMA2. T.R. was awarded a Research Scholarship within the Grant Post Doctoral Fellowship year 2018-Fondazione Umberto Veronesi. The funders had no role in study design; in the collection, analysis, and interpretation of data; in the writing of the report; and in the decision to submit the article for publication. We are extremely 
grateful to Benedetto Terracini and Carlotta Buzzoni for many useful discussions and revision of the manuscript.

\section{CONFLICTS OF INTEREST}

The authors have no potential conflicts of interest to disclose

\section{References}

1 Gupta S, Wilejto M, Pole JD, Guttmann A, Sung L. Low socioeconomic status is associated with worse survival in children with cancer: a systematic review. PLoS One. 2014;9.

2Syse A, Lyngstad TH, Kravdal O. Is mortality after childhood cancer dependent on social or economic resources of parents? A population based study. Int J Cancer. 2012;130:1870-1878.

3Mogensen H, Modig K, Tettamanti G, Talbäck M, Feychting M. Socioeconomic differences in cancer survival among Swedish children. Br J Cancer. 2016;114:118-124.

4Simony SB, Lund LW, Erdmann F, et al. Effect of socioeconomic position on survival after childhood cancer in Denmark. Acta Oncol. 2016;55:742-750.

5Adam M, Rueegg CS, Schmidlin K, et al. Socioeconomic disparities in childhood cancer survival in Switzerland. Int J Cancer. 2016;138:2856-2866.

6Erdmann F, Winther JF, Dalton SO, et al. 2016(January):1096-1104.

7Erdmann F, Winther JF, Dalton SO, Zeeb H, Krøyer A, Bautz A. Survival from tumours of the central nervous system in Danish children: is survival related to family circumstances . Int $\mathbf{J}$ Cancer. 2018;142:671-680.

8Charalampopoulou A, Petridou E, Spyridopoulos T, et al. An integrated evaluation of socioeconomic and clinical factors in the survival from childhood acute lymphoblastic leukaemia: a study in Greece. Eur J Cancer Prev. 2004;13:397-401.

9Sergentanis T, Dessypris N, Kanavidis P, et al. Socioeconomic status, area remoteness, and survival from childhood leukemia: results from the Nationwide Registry for Childhood Hematological Malignancies in Greece. Eur J Cancer Prev. 2013;22:473-479.

10World Health Organization. International Classification of Diseases for Oncology. 3rd ed. $\left(\begin{array}{lll}\text { ICD } & O & 3\end{array}\right)$. Classification of Diseases.

11Steliarova Foucher E, Stiller C, Lacour B, Kaatsch P. International classification of childhood cancer, third edition. Cancer. 2005;103:1457-1467.

12Isaevska E, Manasievska M, Alessi D, et al. Cancer incidence rates and trends among children and adolescents in Piedmont, 1967-2011. PLoS One. 2017;12.

13Cardano M, Costa G, Demaria M. Social mobility and health in the Turin longitudinal study. Soc Sci Med. 2004;58:1563-1574. 
14Dama E, Pastore G, Mosso ML, et al. Time trends and prognostic factors for survival from childhood cancer: a report from the Childhood Cancer Registry of Piedmont (Italy). Eur J Pediatr. 2006; 165:240-249.

15Zuccolo L, Dama E, Maule MM, Pastore G, Merletti F, Magnani C. Updating long term childhood cancer survival trend with period and mixed analysis: good news from population based estimates in Italy. Eur J Cancer. 2006;42:1135-1142.

16Bland JM, Altman DG. The logrank test. BMJ. 2004;328:1073.

17White IR, Royston P, Wood AM. Multiple imputation using chained equations: issues and guidance for practice. Stat Med. 2011;30:377-399..

18StataCorp. Stata statistical software: Release 13. 2013.

19Roddy E, Mueller S. Late effects of treatment of pediatric central nervous system tumors. J Child Neurol. 2016;31:237-254.

20AIRTUM Working Group, CCM AWG. Italian cancer figures, report 2012: cancer in children and adolescents. Epidemiol Prev. 2013;37(1):1-225.

21Bhatia S. Disparities in cancer outcomes: lessons learned from children with cancer. Pediatr Blood Cancer. 2011;56:994-1002. 